\title{
VÉRIFICATION DES CRITĖRES UTILISÉS POUR DÉTERMINER LES POSITIONS DES BANDES HIVERNALES SUR LA PARTIE MARINE DES ÉCAILLES DE SAUMON ATLANTIQUE (SALMO SALAR L.) ADULTE EN FRANCE ET EN ESPAGNE
}

\author{
J.L. BAGLINIÈRE (1), A.G. NICIEZA (2), F. BRAÑ̃̂̀-VIGIL (2), \\ E. PRÉVOST (1)
}

(1) Laboratoire d'Ecologie Hydrobiologique, Station de Physiologie et d'Ecologie des Poissons - INRA - 65, Route de St Brieuc - 35042 RENNES CEDEX - FRANCE.

(2) Departamento de Biologia de Organismos y Sistemas, Universidad de Oviedo, c/Jesùs Arias de Velasco s/n - 33005 OVIEDO - ESPAÑA.

\section{RÉSUMÉ}

Les critères utilisés pour déterminer la position des hivers marins sur les écailles de Saumon atlantique (Salmo salar) sont réexaminés pour les stocks français et espagnols : 658 écailles de saumon pris sur 24 rivières françaises (saison de pêche 1988) et six rivières espagnoles (saisons de pêche 1984 à 1989) ont été analysées. La première bande hivernale se situe bien à une longueur rétromesurée par régression linéaire simple entre 40 et $60 \mathrm{~cm}$ puisque seulement $2,3 \%$ des poissons avaient une taille en dehors de ces limites. Aucune différence n'apparaît entre les stocks français et espagnols. $24,3 \%$ des saumons français et $48 \%$ des poissons espagnols de deux ans de mer et plus avaient une deuxième bande hivernale marine située à une longueur rétromesurée égale ou supérieure à 1,55 fois celle du premier hiver. $45,2 \%$ des saumons français et $70,8 \%$ des poissons espagnols de 3 ans de mer avaient leur troisième bande hivernale marine située à une longueur rétromesurée égale ou supérieure à 1,2 fois celle du second hiver. Dans les deux derniers cas, les différences entre les deux stocks sont significatives.

Les résultats sont discutés et il est proposé d'adopter des valeurs de 1,4 et de 1,17 pour déterminer respectivement la position du deuxième et du troisième hiver marin car le pourcentage de poissons bien classés augmente fortement dans les stocks français et espagnols.

\section{CHECKING OF CRITERIA USED FOR DETERMINING THE POSITION OF SEA WINTER BAND \\ ON ADULT ATLANTIC SALMON (SALMO SALAR L.) SCALES IN FRANCE AND SPAIN}

\begin{abstract}
The factors used to determine the position of sea winter band on scales were reexamined for the Atlantic salmon (Salmo salar L.) French and Spanish stocks. A total of 658 scales of salmon caught in 24 French (1988 fishing season) and six Spanish (fishing seasons from 1984 to 1989 ) rivers were analysed. The first winter band occur well at a back-calculated length between $40 \mathrm{~cm}$ and $60 \mathrm{~cm}$ estimated by linear regression as only $2.3 \%$ of fishes had a size outside these limits. There is no significant difference between the two stocks. In multi seawinter salmon, $24.3 \%$ of French and $48 \%$ of Spanish fish had a second winter band occurring
\end{abstract}


at a back-calculated length equal or superior to 1.55 time this of the first winter. In three seawinter salmon, $45.2 \%$ of French fish and $70.8 \%$ of Spanish fish three sea-winter old had a third winter band occurring at a back-calculated length equal or superior to 1.2 time those one of the second winter. In the two last cases, there is a significant difference between the two stocks.

The results are discussed and it is proposed to adopt the 1.40 and 1.17 values to determine the position of the second and third winter band as the percentage of well determined fishes increases strongly in the French and Spanish stocks.

\section{INTRODUCTION}

Lors de l'atelier international de lecture d'écailles de Saumon atlantique (Salmo salar L.) tenu à Aberdeen en avril 1984, les critères d'interprétation des écailles de cette espèce ont été harmonisés et des normes pour déterminer l'âge marin ont été fixées comme suit (ANONYME, 1984 ; BAGLINIĖRE, 1985) :

- Est considérée comme premier hiver marin toute bande sombre (circuli resserrés) donnant une longueur rétromesurée du saumon (modèle linéaire simple) comprise entre 40 et 60 centimètres.

- Est considérée comme second hiver marin toute bande sombre donnant une longueur rétromesurée du saumon égale ou supérieure à 1,55 fois celle calculée pour le premier hiver marin.

- Est considérée comme troisième hiver marin toute bande sombre donnant une longueur rétromesurée du saumon égale ou supérieure à 1,2 fois celle estimée pour le second hiver marin.

Un deuxième atelier international de lecture d'écailles de Saumon atlantique s'est réuni à Aberdeen en octobre 1988. II en est ressorti que la valeur 1,55 utilisée pour valider le deuxième hiver marin était trop élevée pour les stocks de Saumon du Royaume-Uni et qu'une valeur de 1,4 était plus applicable. Ainsi, à l'issue de cet atelier, il a été demandé que les représentants de chaque pays réexaminent les normes de détermination de l'âge marin définies lors du premier atelier (ANONYME, 1988).

Ce papier présente les résultats de cette vérification pour les stocks de Saumon français et espagnols.

\section{MATÉRIEL ET MÉTHODES}

La vérification des normes de détermination de l'âge marin du Saumon Atlantique a été faite à partir d'un échantillon de saumons adultes :

- capturés par pêche amateur ou professionnelle durant la saison 1988 sur l'ensemble des rivières françaises fréquentées par l'espèce (BAGLINIĖRE, 1985).

- capturés par pêche à la ligne (pêche au filet interdite) durant les saisons 1984 à 1989 sur six rivières des Asturies qui est la province espagnole la plus riche en saumon (VAUCLIN, 1990).

Cette vérification a concerné au total 658 écailles de saumons adultes âgés de $1+, 2$, $2+$ et 3 ans de mer et répartis sur 30 rivières des deux pays (Tableau I). Le nombre de poissons échantillonnés par rivière a été fonction du nombre total de captures et/ou de la répartition des quatre âges de mer observés par cours d'eau.

La rétromesure des longueurs a été faite à l'aide d'un modèle linéaire simple du type :

$$
\mathrm{L}=\mathrm{L}_{\mathrm{T}} \mathrm{L}_{\mathrm{B}} / \mathrm{Le}
$$

avec $L e=$ Rayon de l'écaille selon son plus grand axe.

$L_{T}=$ Longueur à la fourche $(\mathrm{cm})$.

$L_{B}=$ Distance du centre de l'écaille à la fin de la bande hivernale.

$L=$ Longueur rétromesurée à la fin de l'hiver en $\mathrm{cm}$. 
Dans ce qui suit :

$L_{1}$ sera la longueur du poisson (en $\mathrm{cm}$ ) à la fin du premier hiver marin.

$\mathrm{K}_{2}$ sera le rapport Distance du centre de l'écaille à la fin de la seconde bande hivernale marine/Distance du centre de l'écaille à la fin de la première bande hivernale marine.

$\mathrm{K}_{3}$ sera le rapport Distance du centre de l'écaille à la fin de la troisième bande hivernale marine/Distance du centre de l'écaille à la fin de la seconde bande hivernale marine.

Tableau I : Nombre de Saumons atlantiques, étudiés par âge de mer et par rivière pour la vérification des normes de détermination de l'âge marin dans 24 rivières françaises et 6 espagnoles.

Table I : Number of Atlantic Salmon studied according to sea age and river to check the criteria of sea age determination in 24 French and 6 Spanish rivers.

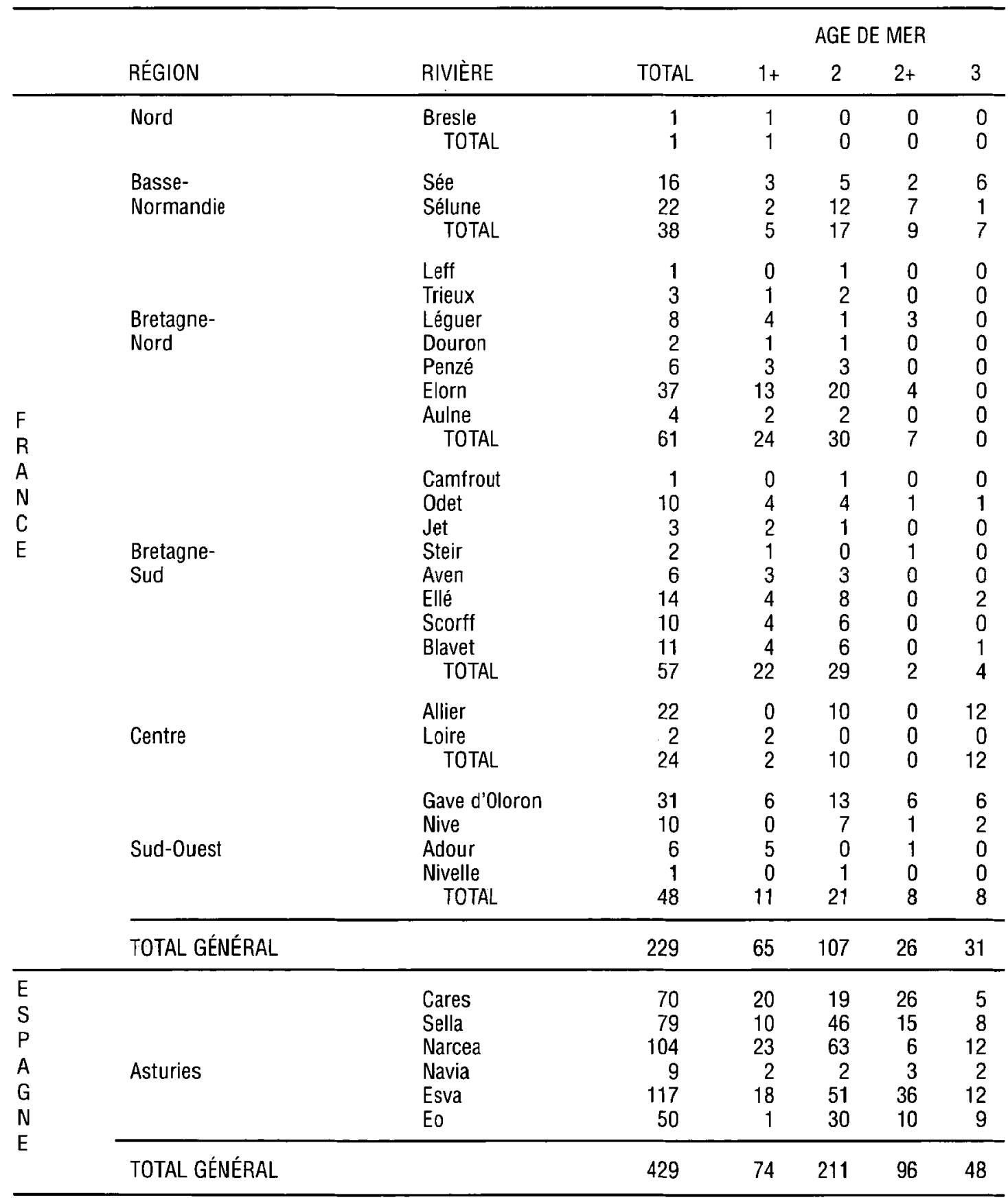




\section{RÉSULTATS}

\subsection{Position de la première bande hivernale $\left(L_{1}\right)$}

L'estimation des longueurs rétromesurées à la fin du premier hiver marin $\left(L_{1}\right)$ a concerné l'échantillon total de saumons adultes. La répartition de ces longueurs rétromesurées a été faite par classe de taille de $1 \mathrm{~cm}$ en relation avec l'âge de mer (Fig. 1). De l'examen des résultats, il ressort que :

- la quasi totalité des poissons ont une longueur $L_{1}$ comprise entre 40 et 60 centimètres : $97,4 \%$ pour les stocks espagnols et $98,3 \%$ pour ceux de France (Fig. 1).

- les poissons de longueur $L_{1}>60 \mathrm{~cm}$ sont proportionnellement plus nombreux que ceux de taille $<40 \mathrm{~cm}$ dans les deux échantillons. Ces poissons concernent les mêmes groupes d'âge de mer quel que soit le stock: $L_{1}>60 \mathrm{~cm}$ groupe d'âge de mer $1+, 2$ et 3 ans, $L_{1}<40 \mathrm{~cm}$ groupe d'âge de mer des 3 ans (Fig. 1).

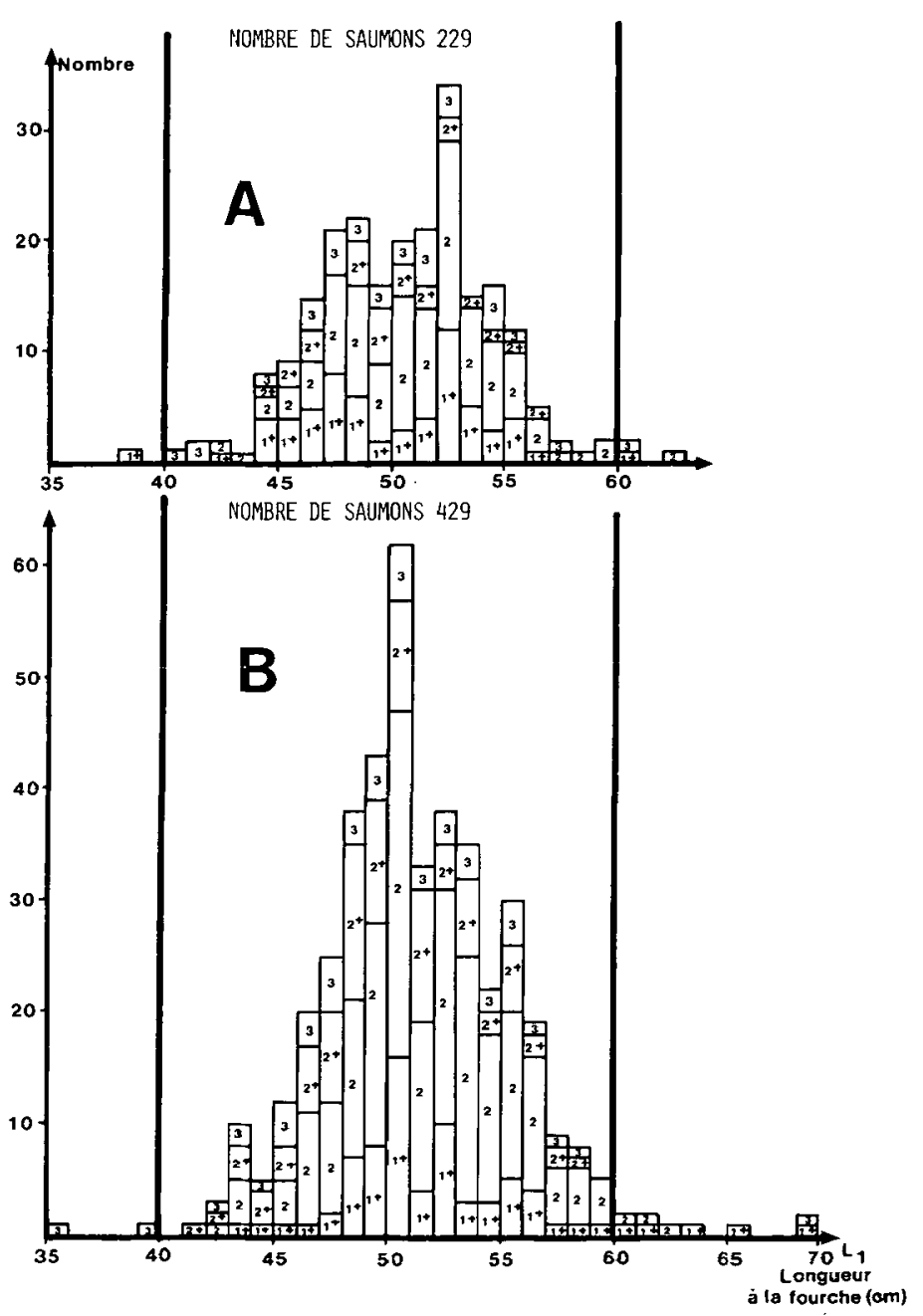

Figure 1 : Distribution de la longueur rétromesurée au premier hiver marin $\left(L_{1}\right)$ chez les saumons atlantiques âgés de 1+, 2, 2+ et 3 ans de mer et échantillonnés sur 24 rivières françaises $(A)$ et 6 espagnoles $(B)$.

$1+, 2,2+, 3=$ Age de mer.

Figure 1 : Distribution of the back calculated length at the first winter band $\left(L_{1}\right)$ in Atlantic Salmon 1+, 2, 2+, 3 sea year old and sampled in 24 French $(A)$ and 6 Spanish (B) rivers.

$1+, 2,2+, 3=$ Sea age. 
- la valeur moyenne de la longueur $L_{1}$ diffère peu dans l'échantillon total par groupe d'âge de mer (Tableau II). Par contre, cette valeur varie beaucoup plus en fonction de la région et de l'âge de mer. Les castillons des rivières des Asturies ont une longueur $L_{1}$ supérieure à celle des poissons des autres régions. Dans les stocks français, cette longueur $L_{1}$ apparaît en général plus élevée chez les saumons du bassin Adour-Gave-Nivelle.

\subsection{Position de la deuxième bande hivernale $\left(K_{2}\right)$}

L'estimation du coefficient $\mathrm{K}_{2}$ a été faite pour 519 saumons adultes (âges de mer 2, 2+ et 3 ans) échantillonnés sur 22 rivières françaises et six espagnoles. L'histogramme de fréquence du coefficient $\mathrm{K}_{2}$ a été tracé en fonction de l'âge de mer (Fig. 2). Les résultats montrent que :

- la majorité des saumons examinés $(68,6 \%: 356 / 519)$ ont un coefficient $K_{2}$ inférieur à 1,55 . Le pourcentage observé dans les stocks français $(75,6 \%: 124 / 164)$ est supérieur $(p<0,01)$ à celui observé pour les stocks espagnols $(62 \%: 220 / 355)$. La répartition des groupes d'âge de mer dans ce sous-échantillon $\left(K_{2}<1,55\right)$ diffère suivant le Pays. Si les proportions des saumons de $2+$ ans de mer ( 72 à $77 \%$ de la classe d'âge) et de 3 ans (58 à $60 \%)$ sont identiques, les poissons de 2 ans de mer sont plus nombreux $(p<0,01)$ dans le sous-échantillon français ( $80 \%$ contre $58 \%$ ). Dans ce dernier, $57 \%$ des poissons de 2 ans de mer ne présentent pas de bande hivernale ou bien montrent seulement un léger resserrement des circuli au bord de l'écaille.

- le groupe de saumons dont le coefficient $K_{2}$ est supérieur ou égal à 1,55 , est constitué en moyenne d'un nombre relativement plus important d'individus de 3 ans de mer, cette observation étant moins nette dans les rivières espagnoles. Néanmoins, ceci confirme la valeur moyenne plus élevée du coefficient $\mathrm{K}_{2}$ de ce groupe d'âge de mer (Tableau II).

- la valeur du coefficient $K_{2}$ apparaît plus élevée et du même ordre pour les poissons de 2 ans et $2+$ ans de mer du Bassin Adour-Gave-Nivelle et de la Région Basse-Normandie. De même cette valeur calculée pour les groupes d'âge de mer $2+$ et 3 ans de la Région des Asturies est très similaire à la moyenne des stocks français (Tableau II).

\subsection{Position de la troisième bande hivernale}

L'estimation du coefficient $K_{3}$ a été réalisée pour 79 saumons de 3 ans de mer échantillonnés sur huit rivières françaises et six espagnoles. L'histogramme de fréquence des valeurs est donné en figure 3. L'examen des résultats montre que:

- la proportion de saumons ayant un coefficient $K_{3} \geq 1,20$ diffère significativement $(p<0,02)$ selon le pays : $45,2 \%(14 / 31)$ pour l'échantillon français et $70,8 \%(34 / 48)$ pour l'échantillon espagnol. Ceci est dû principalement au nombre de poissons français ne présentant pas de bande hivernale ou montrant un léger resserrement des circuli au bord de l'écaille (93,5\% des saumons étudiés) alors que ce phénomène est nettement moins marqué pour les saumons de trois ans de mer des rivières asturiennes.

- la valeur moyenne du coefficient $\mathrm{K}_{3}$ est plus élevée pour les poissons espagnols tandis que dans les stocks français la valeur la plus faible est observée pour les saumons échantillonnés dans le bassin Adour-Gave-Nivelle.

\section{DISCUSSION-CONCLUSION}

De la vérification des normes proposées pour déterminer l'âge marin du Saumon Atlantique des stocks espagnols et français, il ressort que :

- la première bande hivernale marine a bien une position sur l'écaille donnant une longueur rétromesurée du poisson à la fin de l'hiver comprise entre 40 et 60 centimètres,

- la valeur actuelle de 1,55 du coefficient $\mathrm{K}_{2}$ (longueur rétromesurée à la fin du second hiver marin/longueur rétromesurée à la fin du premier hiver marin) paraît trop élevée pour identifier la deuxième bande hivernale sur l'écaille. Ceci est particulièrement valable pour les stocks français et s'explique principalement par la période de remontée de ces poissons située en début d'année (février-avril) et empêchant souvent la formation de la deuxième bande hivernale au bord de l'écaille. Cette hypothèse est en fait confirmée par deux observations: 
Tableau II : Valeurs moyennes de la longueur $L_{1}(e n \mathrm{~cm})$, des coefficients $K_{2}$ et $K_{3}$, par région et par âge de mer chez les saumons adultes échantillonnés dans les rivières françaises et espagnoles.

( ) écart type.

Table II : Mean values of $L_{1}$ length (in $\mathrm{cm}$ ), of $K_{2}$ and $K_{3}$ factors, according to country and sea age in Atlantic Salmon sampled in French and Spanish rivers. () standard deviation.

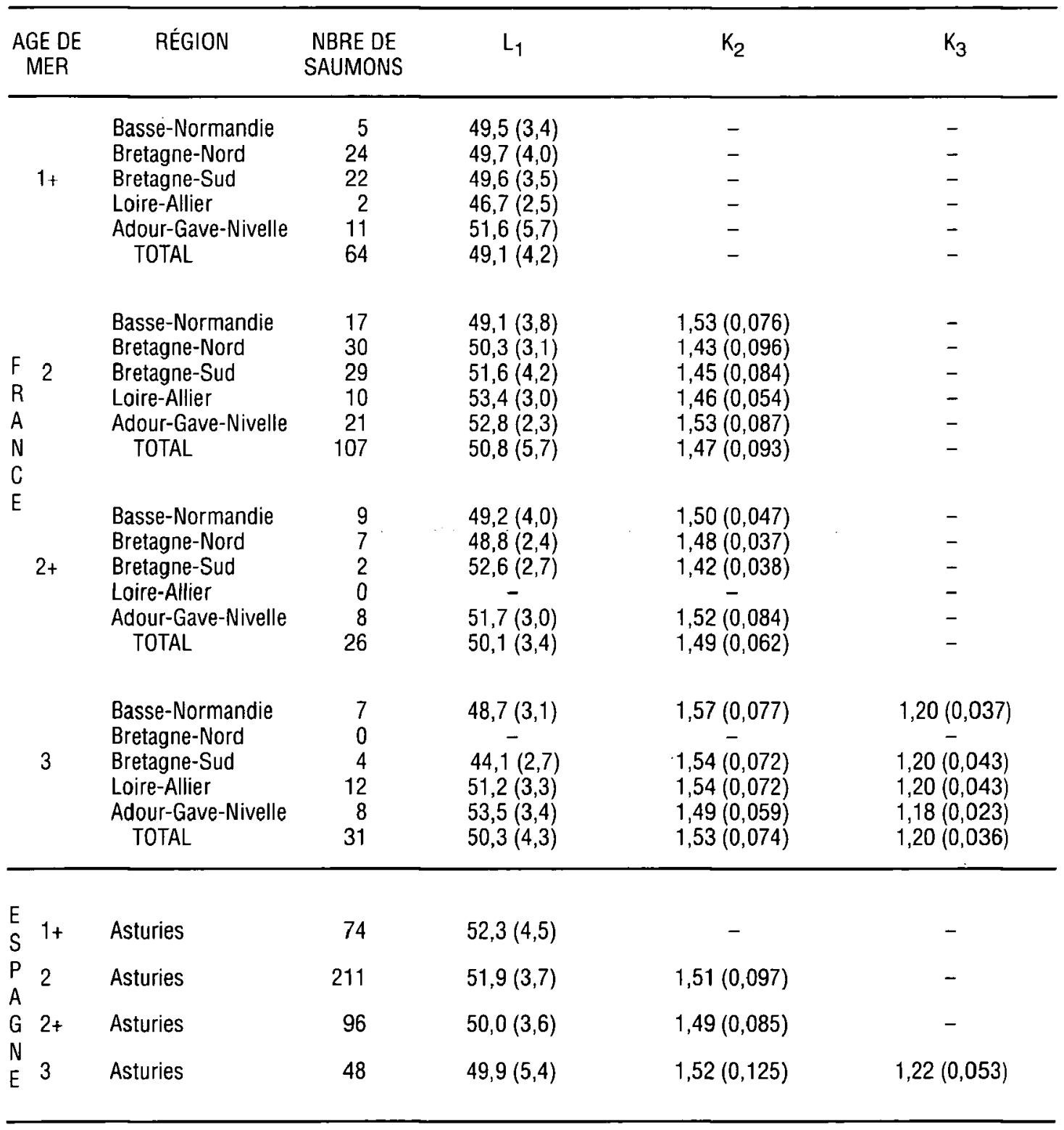

NB : La classification française par région correspond aux unités biogéographiques mises en évidence par PRÉVOST (1987) dans son étude sur les caractéristiques des stocks de saumon français.

- la valeur plus élevée du coefficient $K_{2}$ pour les saumons de 2 ans de mer des stocks espagnols, dont la période de remontée apparaît en moyenne plus tardive qu'en France (avril à juin : VAUCLIN, 1990). deux pays.

- la valeur plus élevée du coefficient $K_{2}$ chez les poissons de 3 ans de mer dans les

Dans les deux cas, la deuxième bande hivernale est complètement formée expliquant ainsi les valeurs plus élevées de $\mathrm{K}_{2}$. 


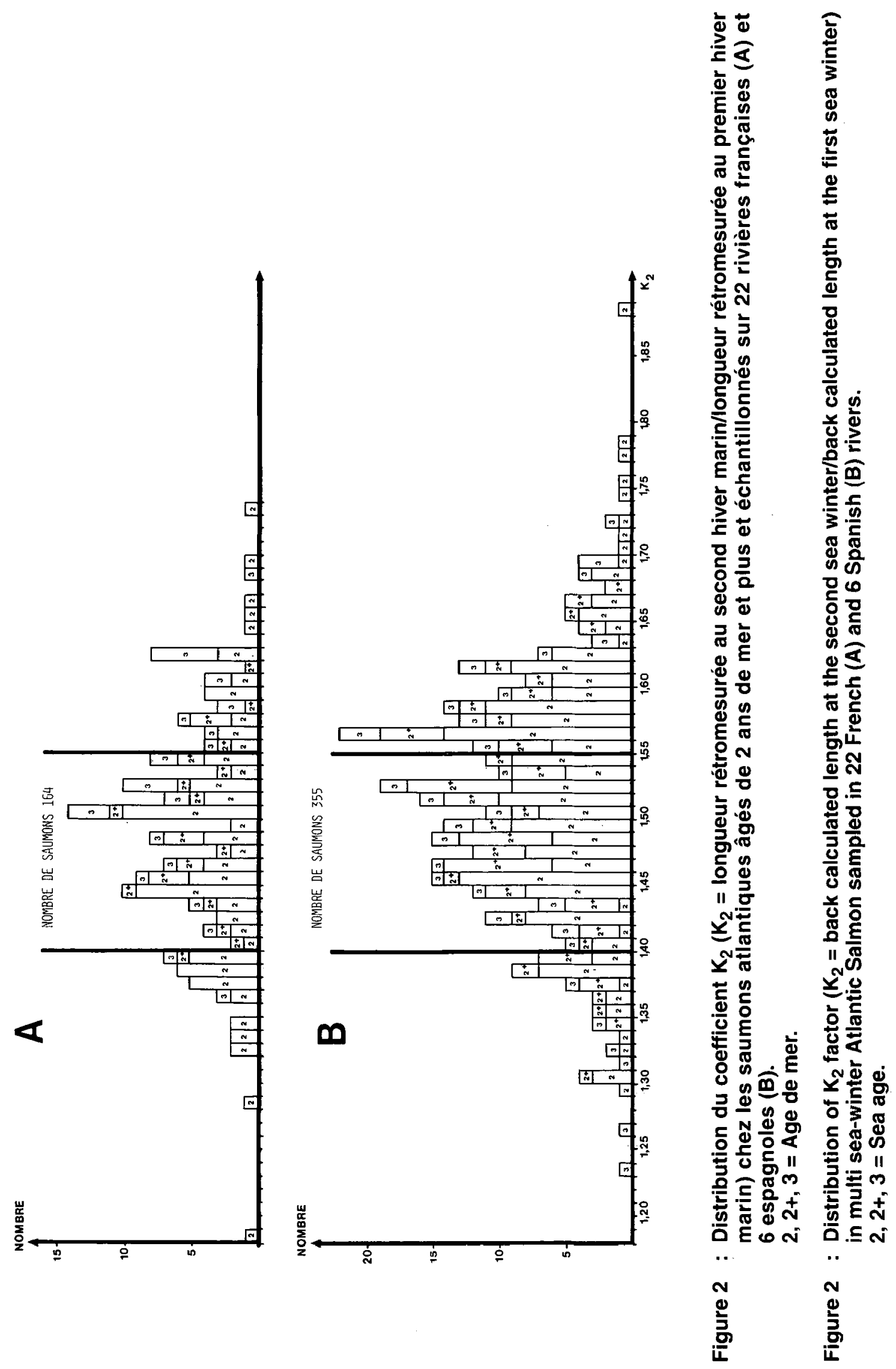


Maintenant si l'on prend 1,4 pour valeur limite de ce coefficient comme cela est déjà fait pour les stocks de saumon du Royaume-Uni (ANONYME, 1988), 83,2 \% des poissons français et $90,4 \%$ des saumons espagnols ont une deuxième bande hivernale correctement positionnée sur l'écaille contre seulement $24,3 \%$ et $48 \%$ respectivement pour la valeur de 1,55 (Fig. 2).

- la valeur 1,2 du coefficient $K_{3}$ (longueur rétromesurée à la fin du troisième hiver marin/longueur rétromesurée à la fin du deuxième hiver marin) semble plus appropriée aux stocks de saumons espagnols qu'à ceux de France pour déterminer sur l'écaille la position de la troisième bande hivernale. Cette différence peut résulter :

- de la structure de l'échantillon. Le groupe des saumons français de 3 ans de mer est constituée en majorité $(64,5 \%)$ d'individus provenant des bassins de l'Allier et de l'Adour. Sur le premier cours d'eau, les saumons remontent en eau douce près de treize mois avant leur reproduction (CUINAT, 1980) et sur le bassin de l'Adour entre janvier et avril (soit en moyenne 9,5 mois avant la fraie) (VIBERT, 1950) alors que ce groupe d'âge de mer migre dans les rivières asturiennes entre les mois de mars et mai (VAUCLIN, 1990). Comme dans le cas des poissons de deux ans de mer, il y a souvent absence de la troisième bande hivernale au bord de l'écaille des poissons de 3 ans de mer en France.

- d'une variabilité importante de la valeur de ce coefficient pour les stocks français.

Maintenant si l'on prend 1,17 pour valeur limite de ce coefficient (valeur choisie en fonction des histogrammes tracés (Fig. 3)), ce qui n'a pas été proposé pour les stocks du Royaume-Uni, le pourcentage de poissons bien classés passe à $87,1 \%$ pour l'échantillon français et à $87,5 \%$ pour les saumons espagnols (Fig. 3 ).
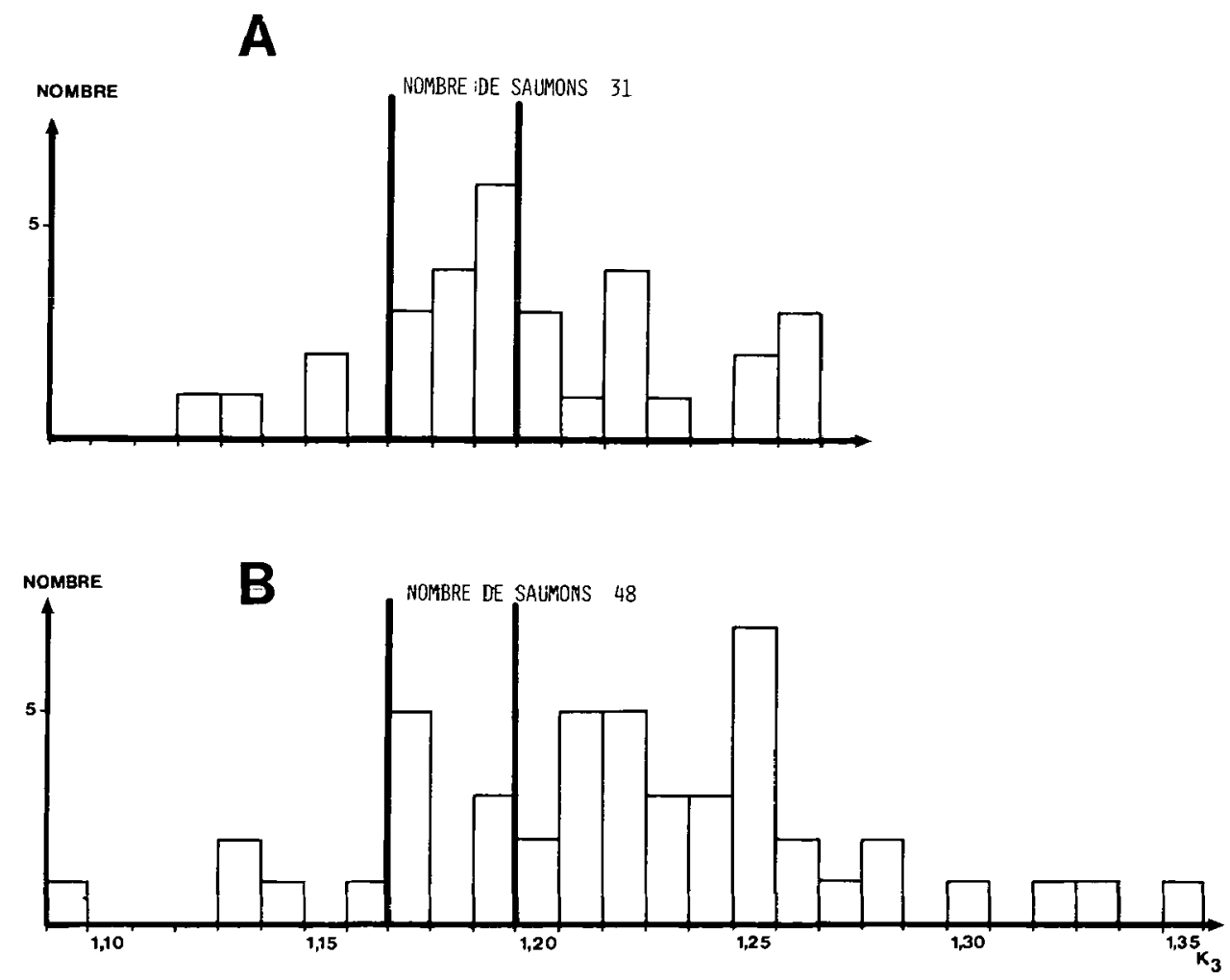

Figure 3 : Distribution du coefficient $\left(K_{3}=\right.$ longueur rétromesurée au troisième hiver marin/longueur rétromesurée au second hiver marin) chez les saumons atlantiques de 3 ans de mer échantillonnés sur 8 rivières françaises $(A)$ et 6 espagnoles (B).

Figure 3 : Distribution of $\mathrm{K}_{3}$ factor $\left(\mathrm{K}_{3}=\right.$ back calculated length at the three sea-winter/back calculated length at the second sea-winter) in three sea-winter Atlantic Salmon sampled in 8 French (A) and 6 Spanish rivers (B). 
En conclusion, si l'on peut retenir le critère de détermination de la première bande hivernale sur l'écaille du saumon adulte (longueur rétromesurée comprise entre 40 et $60 \mathrm{~cm}$ ), les deux autres critères, proposés pour identifier les positions des deuxième et troisième bandes hivernales sur l'écaille du saumon adulte lors du premier atelier international de lecture d'écaille (ANONYME, 1984), paraissent devoir être modifiés comme suit pour les stocks de saumon français et espagnols : coefficient $K_{2} \geq 1,4$ et coefficient $K_{3} \geq 1,17$. De plus les résultats obtenus dans cette étude montrent que la valeur de ces deux derniers coefficients semblent varier suivant les bassins. Enfin, cette vérification des normes a été faite à partir d'écailles pour lesquelles il est impossible de savoir si elles proviennent réellement de la zone de prélèvement standardisée lors de l'atelier international (ANONYME, 1984).

\section{BIBLIOGRAPHIE}

ANONYME, 1984. Atlantic Salmon scale reading. Report of Atlantic Salmon scale reading worshop, Aberdeen, Scotland, 23-28 april 1984, ICES, 1 vol., 17 p.

ANONYME, 1988. Atlantic Salmon scale reading. Report of the second Atlantic Salmon scale reading workshop, Aberdeen, Scotland, 12-14 october 1988, ICES, 1 vol., 16 p.

BAGLINIĖRE J.L., 1985. La détermination de l'âge par scalimétrie chez le saumon atlantique dans son aire de répartition méridionale : utilisation pratique et difficultés de la méthode. Bull. Fr. Pêche Piscic., 298, 69-105.

CUINAT R., 1980. Le saumon du bassin Loire-Allier. Saumons, 34, 26-32.

PRÉVOST E., 1987. Les populations de saumon atlantique (Salmo salar L.) en France : Description ; relation avec les caractéristiques des rivières ; Essai de discrimination. Thèse Doc. Ingénieur, Sci. Agron., ENSA Rennes, 1 vol., $103 \mathrm{p}$.

VAUCLIN V., 1990. Le Saumon Atlantique (Salmo salar) dans la province des Asturies (Espagne) : Eléments de description des populations et de détermination du sexe. Diplôme d'Agronomie Approfondie, Option Halieutique, Ecole Nationale Supérieure Agronomique de Rennes, 1 vol., $37 \mathrm{p}$.

VIBERT R., 1950. Recherches sur le Saumon de l'Adour (Salmo salar L.) (Ages, croissance, cycle génétique, races) 1942-1948. Ann. St. Cent. Hydro. Appl., 3, 27-148. 